\title{
Optimization of Process Parameters for Friction Stir Welding of Dissimilar Aluminium Alloy AA6061 to AA5183 using TOPSIS Technique
}

\author{
Shubham Shukla, Himanshu Shukla
}

\begin{abstract}
Friction stir welding has proven to be the most promising solid state joining process. It can be used to get high weldability in joining of high strength aerospace aluminium alloys and other metallic alloys which used to be low with traditional fusion welding process. This paper emphasises on finding the optimum process parameter for friction stir welding of dissimilar aluminium alloy AA6061 to AA5183 using multi criteria decision making method (MCDM). Friction stir welding was done at different tool rotational speed and transverse velocity and mechanical properties such as tensile strength, percentage elongation and hardness were studied for each weld specimen. Finally optimization was done using TOPSIS (Techniqueof Ordered Preference by Similarity to Ideal Solution). The result revealed that the tool rotational speed of $1200 \mathrm{rpm}$ and welding speed of $80 \mathrm{~mm} / \mathrm{min}$ are the optimum welding parameters.
\end{abstract}

Keywords: Friction stir welding, TOPSIS, Process parameter

\section{INTRODUCTION}

Solid state welding for joining of two dissimilar aluminium alloys has gained a great acceptance in the recent decades. Because of its various advantages over traditional fusion welding used for joining of dissimilar aluminium alloy.

Solid state welding help in overcoming various welding defects like porosity, segregation etc. Joining of two dissimilar metal is a difficult task as both the metal have different chemical composition and mechanical properties. friction stir welding is most successful solid state welding techniquefor this task. In friction stir welding two metal plates are joined by a non consumable rotating -translating tool which has a specially designed pin at its bottom. the shoulder of the tool rubs against the workpiece. Thus the tool help in heating of the workpiece

Revised Manuscript Received on July 25, 2019

Shubham Shukla, Department of Mechanical Engineering, Acropolis Technical Campus Indore ,India.

Himanshu Shukla Department of Mechanical Engineering, Acropolis Technical Campus Indore ,India. to a temperature at which plastic deformation takes place and movement of the material from the leading face of the pin to the trailing face. In friction stir welding the major process parameters are tool geometry, transverse speed and rotational speed of the tool which determine the superiority of weld joint. Hence it is required to select the best welding parameters.

M.V.R.Durga Prasad and Kiran Kumar Namala(1) did the parameter optimization for friction stir welding of dissimilar aluminium alloy AA5083 To AA6061 with process parameters as tool rotational speed, welding speed, and tool tilt angle by using Taguchis approach L9 array. The experimental study showed that the welding speed had the maximum influence on percentage elongation and effect of hardness at weld zone. The tool rotational speed had least influence on the percentage elongation and effect of hardness, the tool tilt angle has major influence and the welding speed has least influence on hardness at HAZ.

M. koilraj, V.Sundereswaran, S. Vijyan did friction stir welding of dissimilar aluminium alloy AA2219 To AA5083 and investigated the optimum process parameter using tTaguchi technique. The parameter considered in were tool rotational speed, transverse speed, tool pin geometry and $\mathrm{D} / \mathrm{d}$ ratio, the result revealed that the optimum welding parameters were tool rotational speed of 700rpm transverse speed of $15 \mathrm{~mm} / \mathrm{min}$ and $\mathrm{D} / \mathrm{d}$ ratio of 3 . the cylindrical thread pin tool profile was found to be the best and the failure occurs at heat affected zone of AA5083. From the above mentioned studies it is clear that a number of research study has been done in parameter optimization in friction stir welding of dissimilar aluminium alloy. but the method of parameter optimization used is taguchi technique which is good for optimization of single objective, but it is not favourable for multi objective optimization. For the optimization of multiple objective, techniques like TOPSIS can be used . In this study friction stir welding of two dissimilar AA6061 to AA5183 is done at different tool rotational speed and transverse velocity. The mechanical properties considered are percentage elongation tensile strength and hardness, 
finally the parameter optimization is done by TOPSOS . In TOPSOS the optimum solution is the one which has smallest Euclidian distance from the ideal solution and largest from the negative ideal solution. it has high consistency, less computation effort and provide more realistic form of. modelling.

\section{Experimental Procedure}

Two plates of AA6061 and AA5183 of thickness 3mm were cut into dimension of 200x100mm using shear cutting machine and were welded by friction stir welding process. The process parameter are shown in the table 1and the weld samples are shown in fig. 1 below.

Table 1 : Process parameters values

\begin{tabular}{|c|c|c|}
\hline $\begin{array}{c}\text { Welded } \\
\text { sample }\end{array}$ & $\begin{array}{c}\text { Tool rotational } \\
\text { speed (rpm) }\end{array}$ & $\begin{array}{c}\text { Tool transverse } \\
\text { speed }\end{array}$ \\
\hline 1 & 1500 & 80 \\
\hline 2 & 1500 & 160 \\
\hline 3 & 1200 & 160 \\
\hline 4 & 900 & 160 \\
\hline 5 & 900 & 80 \\
\hline 6 & 1200 & 120 \\
\hline 7 & 1200 & 80 \\
\hline 8 & 1800 & 80 \\
\hline 9 & 1500 & 120 \\
\hline 10 & 900 & 120 \\
\hline
\end{tabular}

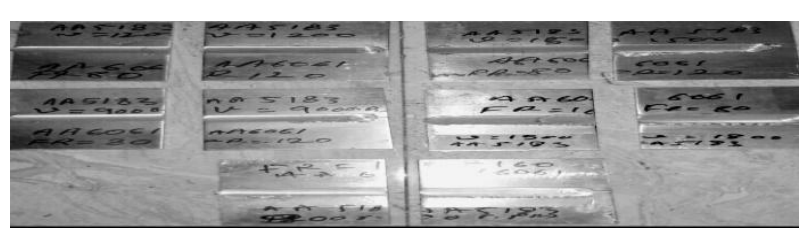

Figure 1: Weld samples after friction stir welding

\section{Tensile test}

Tensile test samples weremade according to ASTM E8, on wire cut EDM machine with dimension of $32 \mathrm{~mm}$ gauge length, $6 \mathrm{~mm}$ width and overall length of $100 \mathrm{~mm}$. The tensile test specimen is shown in the figure below.

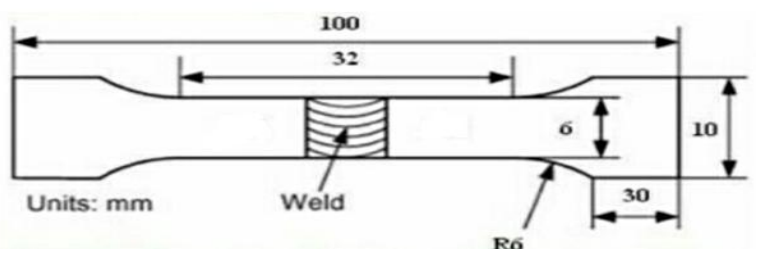

Figure 2: Dimensions of tensile test sample

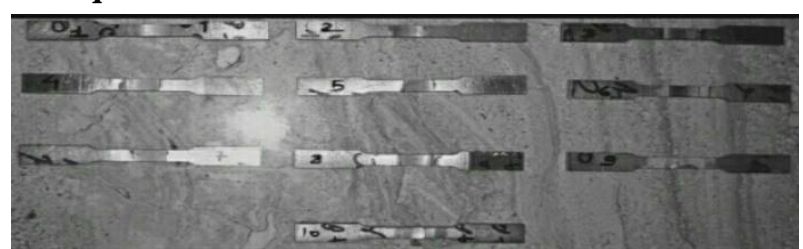

Figure 3 : Tensile test sample before tensile test.

Tensile test was performed to evaluate the values of tensile strength and percentage elongation. The results of the tensile test are shown in the table 2 and graph below.

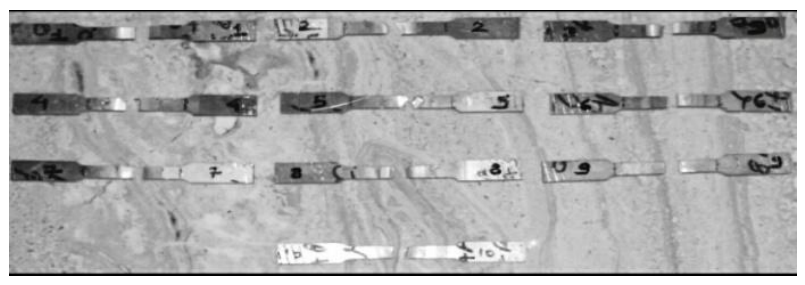

Figure 4: Tensile test samples after tensile test.

Table 2: Tensile strength and percentage elongation values

\begin{tabular}{|c|c|c|}
\hline Weld sample & $\begin{array}{c}\text { Tensile strength } \\
\left(\mathbf{N} / \mathbf{m m}^{\mathbf{2}}\right)\end{array}$ & $\begin{array}{c}\text { Percentage } \\
\text { elongation }\end{array}$ \\
\hline .1 & 142.68 & 7.18 \\
\hline 2 & 172.41 & 12.5 \\
\hline 3 & 178.35 & 12.18 \\
\hline 4 & 59.45 & 6.25 \\
\hline 5 & 130.80 & 16.25 \\
\hline 6 & 178.36 & 10.94 \\
\hline 7 & 148.63 & 12.5 \\
\hline 8 & 178.35 & 13.44 \\
\hline 9 & 166.47 & 8.75 \\
\hline 10 & 160.52 & 11.25 \\
\hline
\end{tabular}

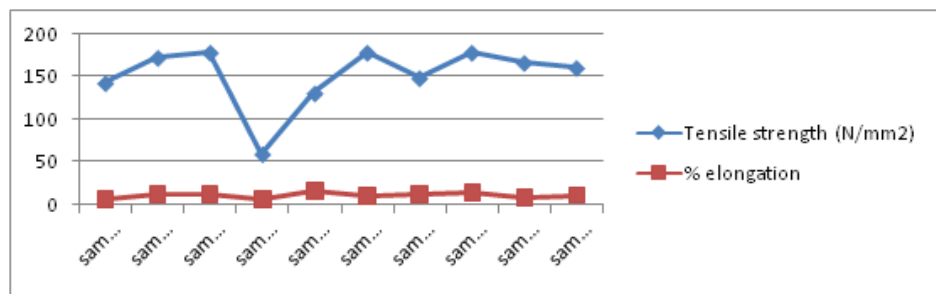

Figure 5: Tensile strength and percentage elongation of weld samples.

\section{Hardness Test}

The weld sample were undergone Vicker's hardness test the result of the test are shown in the table 3 below. 
Table 3: Hardness values.

\begin{tabular}{|c|c|c|}
\hline \multicolumn{2}{|r|}{ Weld sample } & Hardness (HV) \\
\hline \multicolumn{2}{|c|}{1} & 62 \\
\hline \multicolumn{2}{|r|}{2} & 59 \\
\hline \multicolumn{2}{|r|}{3} & 61 \\
\hline \multicolumn{2}{|r|}{4} & 53 \\
\hline \multicolumn{2}{|r|}{5} & 62 \\
\hline \multicolumn{2}{|r|}{6} & 57 \\
\hline \multicolumn{2}{|r|}{7} & 60 \\
\hline \multicolumn{2}{|r|}{8} & 59 \\
\hline \multicolumn{2}{|r|}{9} & 64 \\
\hline \multicolumn{2}{|r|}{10} & 56 \\
\hline \multirow{5}{*}{ 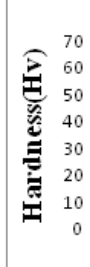 } & \multicolumn{2}{|c|}{ Hardness } \\
\hline & \multicolumn{2}{|c|}{$\rightarrow r^{2}$} \\
\hline & \multicolumn{2}{|c|}{$\begin{array}{r}+2 \\
\end{array}$} \\
\hline & \multicolumn{2}{|c|}{ 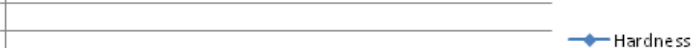 } \\
\hline & & st \\
\hline
\end{tabular}

Figure 6: Hardness of weld sample.

The process parameters were summarized in table 4.

Table 4: Summarized values of process parameters

\begin{tabular}{|c|c|c|c|c|c|}
\hline $\begin{array}{c}\text { Wel } \\
\text { d } \\
\text { sam } \\
\text { ple }\end{array}$ & $\begin{array}{c}\text { Tool } \\
\text { rotati } \\
\text { onal } \\
\text { speed } \\
\text { (rpm) }\end{array}$ & $\begin{array}{c}\text { Tool } \\
\text { transv } \\
\text { erse } \\
\text { speed }\end{array}$ & $\begin{array}{l}\text { Tensi } \\
\text { le } \\
\text { stren } \\
\text { gth } \\
(\mathrm{N} / \mathrm{m} \\
\left.\mathrm{m}^{2}\right)\end{array}$ & $\begin{array}{l}\text { Percen } \\
\text { tage } \\
\text { elonga } \\
\text { tion }\end{array}$ & $\begin{array}{c}\text { Hard } \\
\text { ness } \\
(\mathrm{HV})\end{array}$ \\
\hline 1 & 1500 & 80 & $\begin{array}{c}142.6 \\
8\end{array}$ & 7.18 & 62 \\
\hline 2 & 1500 & 160 & $\begin{array}{c}172.4 \\
1\end{array}$ & 12.5 & 59 \\
\hline 3 & 1200 & 160 & $\begin{array}{c}178.3 \\
5\end{array}$ & 12.18 & 61 \\
\hline 4 & 900 & 160 & 59.45 & 6.25 & 53 \\
\hline 5 & 900 & 80 & $\begin{array}{c}130.8 \\
0\end{array}$ & 16.25 & 62 \\
\hline 6 & 1200 & 120 & $\begin{array}{c}178.3 \\
6\end{array}$ & 10.94 & 57 \\
\hline 7 & 1200 & 80 & $\begin{array}{c}148.6 \\
3\end{array}$ & 12.5 & 60 \\
\hline 8 & 1800 & 80 & $\begin{array}{c}178.3 \\
5\end{array}$ & 13.44 & 59 \\
\hline 9 & 1500 & 120 & $\begin{array}{c}166.4 \\
7\end{array}$ & 8.75 & 64 \\
\hline 10 & 900 & 120 & $\begin{array}{c}160.5 \\
2\end{array}$ & 11.25 & 56 \\
\hline
\end{tabular}

\section{TOPSIS}

The following steps explain the procedure followed as follows.

STEP 1: Normalization of the values by using the equation 1 , table 5 shows the values square summation square root and table 6 shows normalized decision matrix.

\section{Equation 1}

$$
n_{i j_{-}}=a_{i j} / \sqrt{\sum_{i=1}^{\mathrm{m}} a_{i j}^{2}}
$$

wherei=1,2,3...10 and $\mathrm{j}=1,2$ and 3. ( $\mathrm{i}=$ No of sample, $\mathrm{j}=$ values obtained in each mechanical test, $a_{i j}=\mathrm{i}^{\text {th }}$ value of $\mathrm{j}^{\text {th }}$ experimental run.)

Table 5: values of squared summation and square root

\begin{tabular}{|c|c|c|c|}
\hline $\begin{array}{c}\text { weld } \\
\text { sample }\end{array}$ & $\begin{array}{c}\text { Tensile } \\
\text { strength } \\
\left(\mathrm{N} / \mathrm{mm}^{2}\right)\end{array}$ & $\begin{array}{c}\text { Percentage } \\
\text { elongation }\end{array}$ & $\begin{array}{c}\text { Hardness } \\
(\mathrm{HV})\end{array}$ \\
\hline 1 & 142.68 & 7.18 & 62 \\
\hline 2 & 172.41 & 12.5 & 59 \\
\hline 3 & 178.35 & 12.18 & 61 \\
\hline 4 & 59.45 & 6.25 & 53 \\
\hline 5 & 130.8 & 16.25 & 62 \\
\hline 6 & 178.36 & 10.94 & 57 \\
\hline 7 & 148.63 & 12.5 & 60 \\
\hline 8 & 178.35 & 13.44 & 59 \\
\hline 9 & 166.47 & 8.75 & 64 \\
\hline 10 & 160.52 & 11.25 & 56 \\
\hline $\begin{array}{c}\text { sum of } \\
\text { square }\end{array}$ & 241725.3 & 1318.972 & 35261 \\
\hline $\begin{array}{c}\text { square } \\
\text { root }\end{array}$ & 491.6556 & 36.31765 & 187.7791 \\
\hline
\end{tabular}

Published By: 
Optimization of Process Parameters for Friction Stir Welding Of Dissimilar Aluminium Alloy AA6061 to AA5183 Using TOPSIS Technique

Table 6: Normalized decision matrix

\begin{tabular}{|c|c|c|}
\hline $\begin{array}{c}\text { Tensile strength } \\
\left(\mathbf{N} / \mathbf{m m}^{\mathbf{2}}\right)\end{array}$ & $\begin{array}{c}\text { Percentage } \\
\text { elongation }\end{array}$ & $\begin{array}{c}\text { Hardness } \\
(\mathbf{H V})\end{array}$ \\
\hline 0.290203 & 0.1977 & 0.330175 \\
\hline 0.350672 & 0.344185 & 0.314199 \\
\hline 0.362754 & 0.335374 & 0.32485 \\
\hline 0.120918 & 0.172093 & 0.282246 \\
\hline 0.26604 & 0.447441 & 0.330175 \\
\hline 0.362774 & 0.301231 & 0.919355 \\
\hline 0.302305 & 0.344185 & 0.967742 \\
\hline 0.362754 & 0.370068 & 0.314199 \\
\hline 0.338591 & 0.24093 & 0.340826 \\
\hline 0.326489 & 0.309767 & 0.298223 \\
\hline
\end{tabular}

Table 8: Ideal best and Ideal worst values

\begin{tabular}{|c|c|c|c|}
\hline solution & $\begin{array}{c}\text { Tensile strength } \\
\left(\mathbf{N} / \mathbf{m m}^{\mathbf{2}}\right)\end{array}$ & $\begin{array}{c}\text { Percentage } \\
\text { elongation }\end{array}$ & $\begin{array}{c}\text { Hardness } \\
(\mathbf{H V})\end{array}$ \\
\hline $\begin{array}{c}\text { Ideal best }( \\
\left.\mathrm{K}_{\mathrm{j}}^{+}\right)\end{array}$ & 0.108832 & 0.134232 & 0.290323 \\
\hline $\begin{array}{c}\text { Ideal } \\
\text { worst }\left(\mathrm{K}^{-}\right. \\
\mathrm{j})\end{array}$ & 0.036275 & 0.051628 & 0.089467 \\
\hline
\end{tabular}

STEP 4: Euclidian distance was calculated from ideal best and ideal worst solution using the equation 5,6.as shown in table 9

STEP 2: Weight normalized decision matrix was made using equation 2 as shown in table 7 and the weights are assigned to each value so that their sum is 1 ...Hence the value of the assigned weight is 0.333 .

Equation 2

$$
r_{i j}=w_{j} \cdot n_{i j}
$$

Table 7: Weight normalized decision matrix

\begin{tabular}{|c|c|c|}
\hline $\begin{array}{c}\text { Tensile strength } \\
\left(\mathbf{N} / \mathbf{m m}^{\mathbf{2}}\right)\end{array}$ & $\begin{array}{c}\text { Percentage } \\
\text { elongation }\end{array}$ & Hardness (HV) \\
\hline 0.087061 & 0.05931 & 0.099053 \\
\hline 0.105202 & 0.103256 & 0.09426 \\
\hline 0.108826 & 0.100612 & 0.097455 \\
\hline 0.036275 & 0.051628 & 0.084674 \\
\hline 0.079812 & 0.134232 & 0.099053 \\
\hline 0.108832 & 0.090369 & 0.275806 \\
\hline 0.090692 & 0.103256 & 0.290323 \\
\hline 0.108826 & 0.11102 & 0.09426 \\
\hline 0.101577 & 0.072279 & 0.102248 \\
\hline 0.097947 & 0.09293 & 0.089467 \\
\hline
\end{tabular}

.STEP 3: Ideal best $\left(\mathrm{K}^{+}\right)$andthe idealworst $\left(\mathrm{K}^{-}\right)$values were determined using equation 3 as shown in table8.

\section{Equation 3}

$\boldsymbol{K}^{+}=\left\{\operatorname{Max}\left(\boldsymbol{r}_{\boldsymbol{i j}}\right)|\boldsymbol{j} \in \mathrm{J}| \mathrm{i}=1,2,3 \ldots 9\right\}$

\section{Equation 4}

$\mathrm{K}^{-}=\left\{\min \left(\boldsymbol{r}_{\boldsymbol{i} j}\right)|\boldsymbol{j} \in \mathrm{J}| \mathrm{i}=1,2,3 \ldots 9\right\} \quad \mathrm{J}=$ Beneficial attributes

equation 7 and ranking is done, as shown in table 


\section{Equation 7}

$X_{i}=s^{-} /\left(s^{-}-s^{+}\right)$

Table 10: Values of performance score and ranking

\begin{tabular}{|c|c|c|}
\hline $\begin{array}{c}\text { Wel } \\
\mathrm{d} \\
\text { samp } \\
\text { le }\end{array}$ & Performance score & Rank \\
\hline 1 & 0.201878 & \\
\hline 2 & 0.30287 & 10 \\
\hline 3 & 0.309867 & 7 \\
\hline 4 & 0.799364 & 6 \\
\hline 5 & 0.326689 & 4 \\
\hline 6 & 0.815107 & 2 \\
\hline 7 & 0.856584 & 1 \\
\hline 8 & 0.322274 & 5 \\
\hline 9 & 0.260144 & 9 \\
\hline 10 & 0.265493 & 8 \\
\hline
\end{tabular}

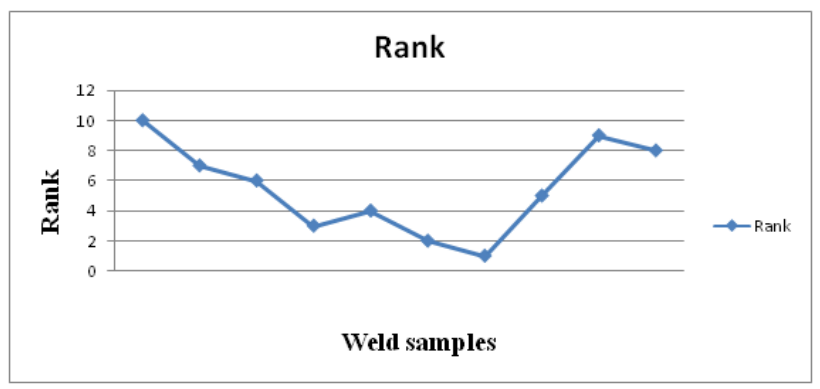

Figure 7: Ranking of weld sample .

\section{CONCLUSION}

The experimental study concluded that the weld sample 7 with a tool rotational speed of $1200 \mathrm{rpm}$,transverse speed of $80 \mathrm{~mm} / \mathrm{min}$, tensile strength of $148.63\left(\mathrm{~N} / \mathrm{mm}^{2}\right)$, percentage elongation of 12.5 , and hardness of $60 \mathrm{Hv}$ had optimum values of process parameters and mechanical properties.

\section{REFERENCES}

1) 5. Prasad M, kumar Namala K. Process Parameters Optimization in Friction Stir Welding by ANOVA. Materials Today: Proceedings. 2018; 5(2):4824-4831.

2) 2. Koilraj M, Sundareswaran V, Vijayan S, Koteswara Rao S. Friction stir welding of dissimilar aluminium alloys AA2219 to AA5083 - Optimization of process parameters using Taguchi technique. Materials \& Design. 2012; 42:1-7.

3) 6. Sudhagar S, Sakthivel M, Mathew P, Daniel S. A multi criteria decision making approach for process improvement in friction stir welding of aluminium alloy. Measurement. 2017;108:1-8..

4) 3. Chien C, Lin W, Chen T. Optimal FSW process parameters for aluminum alloys AA5083. Journal of the Chinese Institute of Engineers. 2011;34(1):99-105.

5) 7. Çalışkan H, Kurşuncu B, Kurbanoğlu C, Güven Ş. Material selection for the tool holder working under hard milling conditions using different multi criteria decision making methods. Materials \& Design. 2013;45:473-479.

6) 8. Mishra R, Ma Z. Friction stir welding and processing. Materials Science and Engineering: R: Reports. 2005;50(1-2):1-78.

7) 9. Shojaeefard M, Behnagh R, Akbari M, Givi M, Farhani F. Modelling and Pareto optimization of mechanical properties of friction stir welded AA7075/AA5083 butt joints using neural network and particle swarm algorithm. Materials \& Design. 2013;44:190-198.

8) 10. Krohling R, Pacheco A. A-TOPSIS - An Approach Based on TOPSIS for Ranking Evolutionary Algorithms. Procedia Computer Science. 2015;55:308-317.

9) 11. Shukla A, Agarwal P, Rana R, Purohit R. Applications of TOPSIS Algorithm on various Manufacturing Processes: A Review. Materials Today: Proceedings. 2017;4(4):5320-5329.

10) 12. Graff W, Sargent D. A new grain-boundary etchant for aluminum alloys. Metallography. 1981;14(1):69-72. 